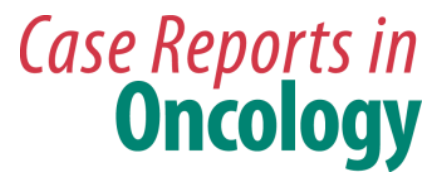

Case Rep Oncol 2018;11:711-720

\title{
Factors Affecting Therapeutic Effects in 17 Patients with Advanced Hepatocellular Carcinoma Who Were Treated with Sorafenib for More than 12 Months
}

\author{
Kazuhiro Katayama ${ }^{a}$ Ryosuke Kiyota ${ }^{a}$ Toshihiro Imai ${ }^{a} \quad$ Yutaro Abe $^{a}$ \\ Tadatoshi Nawa $^{a}$ Hiroshi Wadab Kazuyoshi Ohkawa ${ }^{a}$ \\ aDepartment of Hepato-Biliary and Pancreatic Oncology, Osaka International Cancer \\ Institute, Osaka, Japan; bDepartment of Surgery, Osaka International Cancer Institute, \\ Osaka, Japan
}

\section{Keywords}

Hepatocellular carcinoma · Sorafenib · Adverse events · Long-term survival

\begin{abstract}
Background and Objective: Sorafenib is recommended for treating advanced hepatocellular carcinoma. However, it is frequently discontinued because of adverse events, which greatly affects its therapeutic effects. Furthermore, because patients treated with sorafenib for a long period can presumably tolerate adverse events, this study aimed to identify their characteristics and analyze factors affecting the therapeutic effects of the drug. Subjects and Methods: Seventeen patients with hepatocellular carcinoma who received sorafenib for 12 months or longer at our hospital between January 2009 and October 2015 were included. In these 17 patients, factors affecting the time to untreatable progression were analyzed using a Cox proportional hazards model, Kaplan-Meier curve, and log-rank test. Results: In the 17 patients, the mean
\end{abstract}


sorafenib dose was $433 \mathrm{mg} /$ day. The drug was discontinued in 12 patients, 9 (75\%) of whom discontinued it because of progressive disease. The median time to untreatable progression was 23.1 months. The contributors to favorable therapeutic effects included administration of at least two sessions of concomitant therapy after initiating sorafenib therapy, a low neutrophil-to-lymphocyte ratio, and a decreased total bilirubin level. Conclusion: Achieving favorable therapeutic effects of sorafenib requires strict dose adjustment that allows better control of adverse events and long-term administration of the drug. Furthermore, combining sorafenib with other therapies, a low neutrophil-to-lymphocyte ratio, and a decreased total bilirubin level are useful predictors of favorable effects.

(C) 2018 The Author(s)

Published by S. Karger AG, Basel

\section{Introduction}

Hepatocellular carcinoma (HCC) is the third most common cause of cancer-related death in the world [1], and its management is a major health and welfare challenge. In Europe and the United States, therapeutic strategies for HCC are based on the Barcelona Clinic Liver Cancer staging system, and sorafenib is recommended for treating advanced HCC [2]. The efficacy of sorafenib for advanced HCC has been demonstrated in two randomized controlled trials [3, 4] and subsequently confirmed by several real-world studies [5-7]. Simultaneously, sorafenib-associated problems have also been revealed. Discontinuation of sorafenib is mainly attributed to disease progression and adverse events (AEs) [3-7]. In case of disease progression, which implies sorafenib ineffectiveness, treatment discontinuation is inevitable. Meanwhile, when AEs become severe, the drug must be discontinued even in some patients responding to its antitumor effect. Thus, an important therapeutic strategy is controlling AEs to maximize the antitumor effect of the drug. For several types of AEs, therapeutic outcomes are better in patients with severe symptoms than in those without; therefore, AEs are reportedly a possible predictor for therapeutic outcomes [8]. Since the use of sorafenib started in real-world settings, various measures against AEs have been innovated. Major AEs include hand-foot syndrome, hypertension, diarrhea, malaise, and hepatic dysfunction, for which symptomatic treatments, such as moisturization of the hands and feet, antihypertensive therapy, and administration of antidiarrheal drugs, have been effective $[9,10]$. Moreover, sorafenib becomes more effective through appropriate dose adjustment in patients with hepatic dysfunction [5].

As described above, the number of patients who can receive long-term administration of the drug has increased owing to the implementation of various measures. In patients treated for a short period, it is difficult to determine whether sorafenib is sufficiently effective. On the other hand, in patients who respond to measures against AEs and can be treated with sorafenib for a somewhat longer period, the next challenge is how to achieve its antitumor effect.

However, many previous studies on the outcomes of sorafenib therapy identify factors reducing the incidence of AEs as factors affecting therapeutic outcomes because all patients receiving sorafenib were analyzed [3-7]. Thus, by selecting patients treated for 12 months or longer from all treated patients, study subjects can be limited predominantly to those who responded to measures against AEs. By revealing characteristics of patients receiving longterm sorafenib therapy and identifying factors affecting its therapeutic effects based on those characteristics, methods to improve the effects of sorafenib can be revealed. 


\section{Case Reports in Oncology}

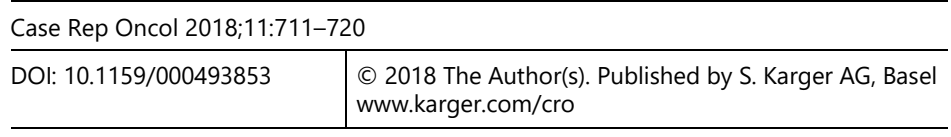

Katayama et al.: Factors Affecting Therapeutic Effects in 17 Patients with Advanced Hepatocellular Carcinoma Who Were Treated with Sorafenib for More than 12 Months

\section{Subjects and Methods}

\section{Subjects}

Of 130 consecutive patients treated for HCC with sorafenib at our hospital between January 2009 and October 2015, 17 patients, accounting for 13.1\%, were selected via a search for patients treated for at least 12 months (including continuous and noncontinuous treatment) in electronic medical record data. The characteristics of the 17 patients at the start of sorafenib therapy are shown in Table 1.

\section{Methods}

In these patients, clinical course and factors affecting time to untreatable progression (TTUP) were retrospectively analyzed. Clinical course variables included TTUP, duration of sorafenib therapy, mean dose during the treatment period, and administration of other therapies. Factors affecting TTUP included age, sex, body mass index, Child-Pugh classification, neutrophil-to-lymphocyte ratio (NLR), blood albumin concentration, blood bilirubin concentration, prothrombin time, hemoglobin level, platelet count, alpha-fetoprotein (AFP) level, AFP-L3 fraction, protein induced by vitamin-K antagonist-II (PIVKA-II), initial sorafenib dose, period when sorafenib was actually administered/period after the start of sorafenib therapy, mean dose, and frequency of sorafenib administration after treatment initiation.

\section{Statistical Analysis}

Factors affecting the therapeutic effects of sorafenib (TTUP) were analyzed using a univariate Cox proportional hazards model. Although multivariate analysis was not performed because of the small sample size, factors with a $p$ value $\leq 0.10$ were additionally analyzed using a Kaplan-Meier curve and log-rank test.

\section{Results}

\section{Sorafenib Therapy}

Data on sorafenib therapy in the study patients are as follows: The median TTUP was 23.1 months (range, 11.9-50.8). During this time, the median period of sorafenib administration was 13.9 months, and the median administration rate (dosing period/treatment period $\times 100$ ) was $87.5 \%$. The mean daily dose of sorafenib was $433 \mathrm{mg} /$ day. The median times of additional treatments sessions were 2 (range, 0-7). Additional treatments included radiofrequency ablation therapy, transarterial chemoembolization, transarterial infusion of anti-cancer agents, or radiation therapy.

The therapy was discontinued permanently in 12 of the 17 patients, and the reasons for treatment discontinuation were progressive disease (PD) in nine patients and hepatic dysfunction in three patients. One of these three patients developed hepatic encephalopathy immediately after the daily dose was increased from 400 to $800 \mathrm{mg}$, and the therapy was discontinued. 


\section{Case Reports in Oncology} \begin{tabular}{l|l} 
Case Rep Oncol 2018;11:711-720 \\
\hline DOI: 10.1159/000493853 & $\begin{array}{l}\text { (c) } 2018 \text { The Author(s). Published by S. Karger AG, Basel } \\
\text { www.karger.com/cro }\end{array}$
\end{tabular}

Katayama et al.: Factors Affecting Therapeutic Effects in 17 Patients with Advanced Hepatocellular Carcinoma Who Were Treated with Sorafenib for More than 12 Months

\section{Contributors to TTUP}

Based on the investigation of contributors to TTUP in sorafenib therapy (Table 2), administration of two sessions or more of additional therapies combined with sorafenib therapy was a contributor to favorable outcomes. Furthermore, NLR being lower than the median and a total bilirubin level $<1.0 \mathrm{mg} / \mathrm{dL}$ before sorafenib therapy contributed to favorable outcomes, although their impact was marginal. When these three contributors were analyzed using a Kaplan-Meier curve, a log-rank test revealed significant differences in all three contributors (Fig. 1).

\section{Discussion}

Patients receiving sorafenib for 12 months or longer accounted for $13.1 \%$ of those receiving the drug at our hospital during the study period. This ratio is almost comparable with that $(10.2 \%)$ calculated from the data from the postmarketing surveillance of sorafenib $(1,119$ cases) in Japan [6]. Based on this, the characteristics of the subjects of the present study is likely to be nearly comparable with the actual proportion of patients receiving sorafenib for 12 months or longer throughout Japan. The study results may thus provide reference for future sorafenib therapy.

Because this study included only patients receiving sorafenib for 12 months or longer, adverse reactions commonly observed in the early stages of treatment were overcome in these patients. The reason for discontinuing sorafenib was PD in $75 \%$ of patients who discontinued it. This proportion greatly differs from the frequency of this reason for discontinuation reported in previous studies on sorafenib. In the Sorafenib Hepatocellular Carcinoma Assessment Randomized Protocol Trial, the reasons for discontinuing sorafenib were AEs in 86/226 patients $(38.1 \%)$ and PD in $61 / 226$ patients (27.0\%) [3]. Postmarketing surveillance in Italy showed that the frequency of the reasons for discontinuing sorafenib was $56 \%$ for AEs and hepatic dysfunction and 44\% for PD [5], and postmarketing surveillance in Japan showed that the frequency was $44.5 \%$ for AEs and $33.8 \%$ for PD [6]. A possible reason for the higher proportion of patients who discontinued sorafenib because of PD in this study was that many patients discontinue sorafenib because of AEs during the first year of treatment. This study excluded such patients and included those who could tolerate sorafenib-associated AEs. Another possible reason is that the mean daily dose of sorafenib in this study was $433 \mathrm{mg}$, which was almost half of the recommended dose, suggesting that attending physicians might have become familiar with measures against sorafenib-associated AEs and thus adjusted the doses before the drug would be discontinued because of AEs. The postmarketing surveillance in Italy also showed that overall survival (OS) was better in patients receiving a half dose than in those receiving a full dose according to the mean daily dose [5]. To prevent discontinuation of sorafenib owing to AEs or hepatic dysfunction, strict dose adjustment may be important.

In addition, the absence of extrahepatic metastasis, absence of macroscopic vascular invasion (MVI), performance status (PS) 0-1, and favorable hepatic reserve were identified as contributors to favorable outcomes in previous studies on sorafenib therapy [3-7]. Because the present study is a retrospective study, data on PS were unavailable. Meanwhile, hepatic reserve was graded as Child-Pugh $A$ in 13 of the 17 patients, confirming that the initiation of sorafenib therapy is an important determinant of outcomes in patients with favorable hepatic 


\section{Case Reports in Oncology}

Case Rep Oncol 2018;11:711-720

DOI: $10.1159 / 000493853$

(c) 2018 The Author(s). Published by S. Karger AG, Base www.karger.com/cro

Katayama et al.: Factors Affecting Therapeutic Effects in 17 Patients with Advanced

Hepatocellular Carcinoma Who Were Treated with Sorafenib for More than 12 Months

reserve. However, inclusion of five patients with MVI (stage IVa) and six patients with extrahepatic metastasis (stage IVb) among the 17 patients suggests that long-term administration of sorafenib may be feasible even in such patients with advanced HCC.

Recent studies have indicated that a low NLR contributes to favorable outcomes of sorafenib therapy [7]. Because the median NLR of 2.00 in the present study is lower than the previously reported median NLR of 3.00 [7], long-term administration of sorafenib may likely be feasible in patients with a low NLR.

As described above, the patients included in this study were tolerant to AEs such as sorafenib-associated hepatic dysfunction. Sorafenib therapy is associated with two major factors that lead to discontinuation. One is an AE or hepatic dysfunction, and the other is PD. Of them, overcoming the former seems to be the first step of sorafenib therapy. Although the present study confirmed this, we also assessed factors affecting the therapeutic effects of sorafenib to explore measures against PD, in other words, strategies to improve the antitumor effect of the drug, which is the second challenge in sorafenib therapy.

The present study identified administration of two sessions or more of a concomitant therapy after initiating sorafenib therapy to be a significant contributor to favorable outcomes. Interestingly, the Global Investigation of Therapeutic Decisions in Hepatocellular Carcinoma and of Treatment with Sorafenib (GIDEON) also showed that outcomes were significantly better in patients receiving combined sorafenib and transarterial chemoembolization than in those receiving sorafenib monotherapy [11]. Because negative outcomes of combination therapy with sorafenib and transarterial chemoembolization have also been reported [12], further studies are needed to draw conclusions on this issue. Sorafenib basically maintains HCC in the stable disease status to improve survival. Because stable disease includes tumor growth of less than $20 \%$, the condition of patients with tumors growing by $1 \%$ to $19 \%$ deteriorates with a longer duration. Moreover, during sorafenib therapy, development of resistance may result in PD. The antitumor effect of sorafenib alone is less potent. However, if a therapy that enhances its antitumor effect and can be tolerated is added, the therapeutic effect of sorafenib can be further improved.

Recent studies have demonstrated that regorafenib is effective as a second-line treatment after sorafenib therapy as the first-line treatment for advanced HCC [13]. Because regorafenib has actions similar to sorafenib, regorafenib may exert a potent antitumor effect without much impact from AEs by its administration to only patients tolerating sorafenib-associated AEs.

The NLR was identified as a factor affecting outcomes of sorafenib therapy by Bruix et al. [7]. In their study on 827 patients receiving sorafenib, OS was significantly better in those with a median NLR of 3.00 or lower than in those with a median NLR exceeding 3.00. The lower median NLR of 2.00 in all 17 patients included in the present study is compatible with the finding of Bruix et al. Furthermore, because the present study including 17 patients also showed that outcomes were significantly better in those with a median NLR of 2.00 or lower than in those with a median NLR exceeding 2.00, NLR is also a useful prognostic predictor in patients who tolerate AEs and receive long-term administration of sorafenib.

There are several limitations to this study. First, this study was retrospective, and a prospective study that further builds upon these results should be conducted. Second, the sample size was small ( $n=17)$, and the results of this study should be confirmed across a larger number of patients. 


\section{Case Reports in Oncology}

Case Rep Oncol 2018;11:711-720

DOI: $10.1159 / 000493853$

(C) 2018 The Author(s). Published by S. Karger AG, Base www.karger.com/cro

Katayama et al:: Factors Affecting Therapeutic Effects in 17 Patients with Advanced

Hepatocellular Carcinoma Who Were Treated with Sorafenib for More than 12 Months

\section{Conclusion}

Achievement of favorable therapeutic effects of sorafenib requires strict dose adjustment that allows better control of AEs and long-term administration of the drug. Furthermore, combination with other therapies, a low NLR, and a decreased total bilirubin level are useful predictors of favorable effects.

\section{Statement of Ethics}

This study was approved by the ethics committee of our hospital (Approval No. 1706080505). Written informed consent was waived by the Institutional Review Board.

\section{Disclosure Statement}

The authors have no conflict of interest to declare.

\section{Funding Sources}

This study was partly supported by a grant from Seijinnbyo-Yobou Kyokai.

\section{Author Contributions}

Study concept and design, KK; Acquisition of data, RK, TI, YA, TN, and HW; Drafting of the manuscript, KK; Critical revision of the manuscript for important intellectual content, HW, KO; Statistical analysis, KK and TN; All authors approved final version of manuscript.

\section{References}

1 Ferlay J, Soerjomataram I, Dikshit R, Eser S, Mathers C, Rebelo M, et al. Cancer incidence and mortality worldwide: sources, methods and major patterns in GLOBOCAN 2012. Int J Cancer. 2015 Mar;136(5):E35986.

2 Forner A, Llovet JM, Bruix J. Hepatocellular carcinoma. Lancet. 2012 Mar;379(9822):1245-55.

3 Llovet JM, Ricci S, Mazzaferro V, Hilgard P, Gane E, Blanc JF, et al.; SHARP Investigators Study Group. Sorafenib in advanced hepatocellular carcinoma. N Engl J Med. 2008 Jul;359(4):378-90.

4 Cheng AL, Kang YK, Chen Z, Tsao CJ, Qin S, Kim JS, et al. Efficacy and safety of sorafenib in patients in the Asia-Pacific region with advanced hepatocellular carcinoma: a phase III randomised, double-blind, placebocontrolled trial. Lancet Oncol. 2009 Jan;10(1):25-34.

5 Iavarone M, Cabibbo G, Piscaglia F, Zavaglia C, Grieco A, Villa E, et al.; SOFIA (SOraFenib Italian Assessment) study group. Field-practice study of sorafenib therapy for hepatocellular carcinoma: a prospective multicenter study in Italy. Hepatology. 2011 Dec;54(6):2055-63.

6 Kaneko S, Ikeda K, Matsuzaki Y, Furuse J, Minami H, Okayama Y, et al. Safety and effectiveness of sorafenib in Japanese patients with hepatocellular carcinoma in daily medical practice: interim analysis of a prospective postmarketing all-patient surveillance study. J Gastroenterol. 2016 Oct;51(10):1011-21. 
7 Bruix J, Cheng AL, Meinhardt G, Nakajima K, De Sanctis Y, Llovet J. Prognostic factors and predictors of sorafenib benefit in patients with hepatocellular carcinoma: analysis of two phase III studies. J Hepatol. 2017 Nov;67(5):999-1008.

8 Lamarca A, Abdel-Rahman O, Salu I, McNamara MG, Valle JW, Hubner RA. Identification of clinical biomarkers for patients with advanced hepatocellular carcinoma receiving sorafenib. Clin Transl Oncol. 2017 Mar;19(3):364-72.

9 Ren Z, Zhu K, Kang H, Lu M, Qu Z, Lu L, et al. Randomized controlled trial of the prophylactic effect of ureabased cream on sorafenib-associated hand-foot skin reactions in patients with advanced hepatocellular carcinoma. J Clin Oncol. 2015 Mar;33(8):894-900.

10 Izzedine H, Ederhy S, Goldwasser F, Soria JC, Milano G, Cohen A, et al. Management of hypertension in angiogenesis inhibitor-treated patients. Ann Oncol. 2009 May;20(5):807-15.

11 Geschwind JF, Kudo M, Marrero JA, Venook AP, Chen XP, Bronowicki JP, et al. TACE treatment in patients with sorafenib-treated unresectable hepatocellular carcinoma in clinical practice: final analysis of GIDEON. Radiology. 2016 May;279(2):630-40.

12 Kudo M, Imanaka K, Chida N, Nakachi K, Tak WY, Takayama T, et al. Phase III study of sorafenib after transarterial chemoembolisation in Japanese and Korean patients with unresectable hepatocellular carcinoma. Eur J Cancer. 2011 Sep;47(14):2117-27.

13 Bruix J, Qin S, Merle P, Granito A, Huang YH, Bodoky G, et al.; RESORCE Investigators. Regorafenib for patients with hepatocellular carcinoma who progressed on sorafenib treatment (RESORCE): a randomised, double-blind, placebo-controlled, phase 3 trial. Lancet. 2017 Jan;389(10064):56-66. 
(A)
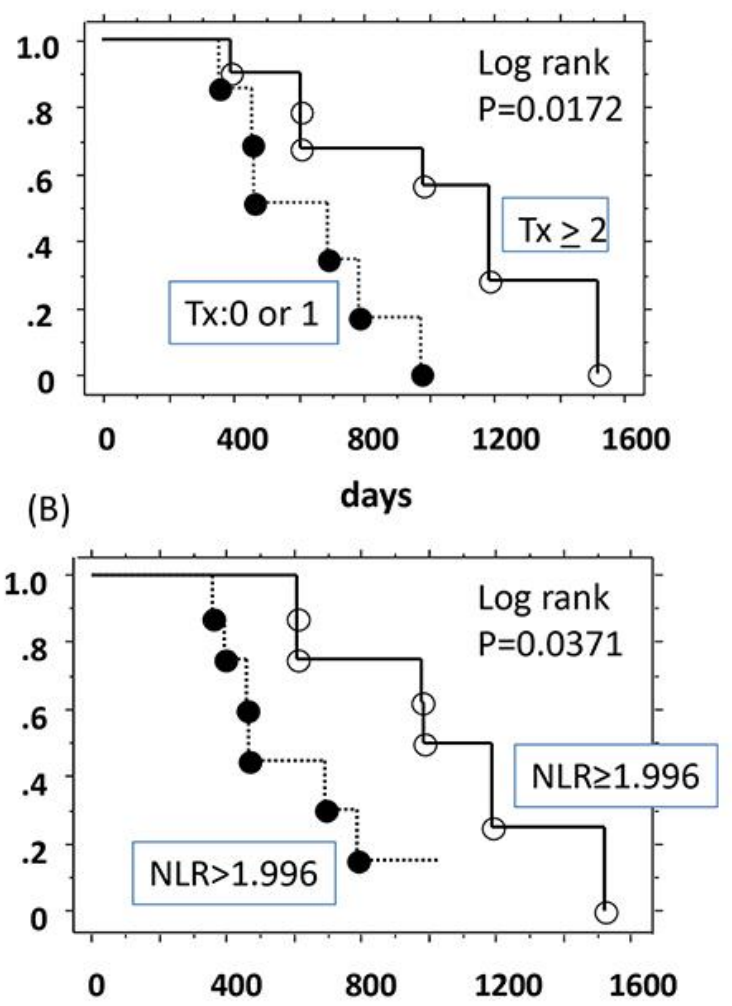

(C)

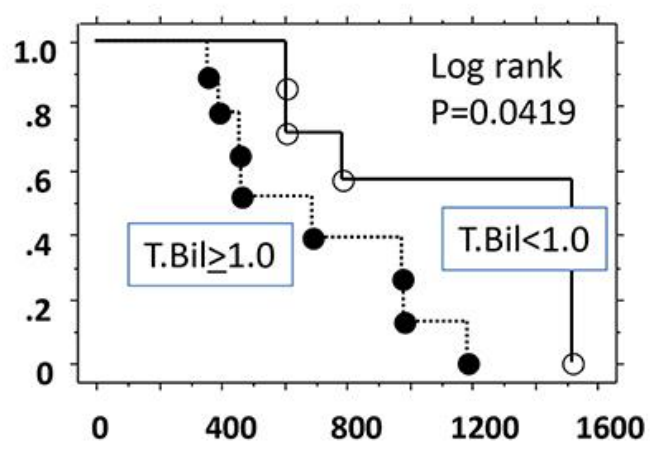

Fig. 1. Kaplan-Meier curve plotting the time to untreatable progression. (A) Combination with other therapies: at least two sessions versus one or no session. (B) Neutrophil-to-lymphocyte ratio: $\leq 2.00$ versus $>2.00$. (C) Total bilirubin level: $\leq 1.0$ versus $>1.0 \mathrm{mg} / \mathrm{dL}$. 


\section{Case Reports in Oncology}

Table 1. Patients' characteristics

\begin{tabular}{ll}
\hline Item & Data \\
\hline Age, years & $67.1 \pm 11.3$ \\
Male:Female & $15: 2$ \\
Body Mass Index & $23.3 \pm 3.7$ \\
HCV/HBV/NBNC & $9 / 3 / 5$ \\
Period between start of HCC treatment and start of sorafenib, days & $858.1 \pm 798.9$ \\
Tumor stage (TNM classification) (II/III/IVa/IVb) & $1 / 5 / 5 / 6$ \\
Child-Pugh classification (A/B/C) & $13 / 4 / 0$ \\
Albumin, g/dL & $3.6 \pm 0.6$ \\
Prothrombin time, \% & $85.1 \pm 18.3$ \\
Total bilirubin, mg/dL & $1.02 \pm 0.52$ \\
AST, U/L & $46.8 \pm 21.5$ \\
ALT, U/L & $33.2 \pm 16.9$ \\
Hb, g/dL & $12.7 \pm 1.2$ \\
NLR & $3.52 \pm 0.56($ median, 2.00$)$ \\
Platelets ( $\left.\times 10^{4} / \mu \mathrm{L}\right)$ & $12.0 \pm 4.9$ \\
Alpha-feto protein, ng/mL & $2,660.5 \pm 6,328.4(3-4,469)$ \\
Protein induced by vitamin-K absence-II, mAU/mL & $3,233.7 \pm 8,427.6(0-7,020)^{\mathrm{b}}$ \\
Initial dose of sorafenib, mg/day $(800 / 600 / 400)$ & $8 / 1 / 8$
\end{tabular}

Values are expressed as mean \pm S.D. HCV, hepatitis C virus; HBV, hepatitis B virus; NBNC, non-B non-C; HCC, hepatocellular carcinoma; AST, aspartate aminotransferase; ALT, alanine aminotransferase; $\mathrm{Hb}$, hemoglobin; NLR, neutrophil-lymphocyte ratio; The range of numbers are shown in parenthesis. TMN, tumor, node and metastasis; a TMN classification based on the criteria of the Liver Cancer Study Group of Japan. b Values of PIVKA-II $<30$ are expressed as 0. 
Table 2. Identification of prognostic factors for time to untreatable progression on univariate analysis

\begin{tabular}{|c|c|c|c|}
\hline Items & Hazard ratio & $95 \% \mathrm{CI}$ & $p$ \\
\hline Age & 0.991 & $0.942-1.044$ & 0.7452 \\
\hline Body mass index ( $\leq 22$ vs. $>22)$ & 0.649 & $0.039-2.540$ & 0.5344 \\
\hline TMN stage (II, III vs. IVa, IVb) & 1.174 & $0.356-3.871$ & 0.7927 \\
\hline Child-Pugh (A vs. B) & 0.516 & $0.128-2.085$ & 0.3531 \\
\hline Albumin ( $\leq 3.5$ vs. $>3.5 \mathrm{~g} / \mathrm{dL})$ & 1.782 & $0.496-6.408$ & 0.3762 \\
\hline Total bilirubin ( $\leq 1.0$ vs. $>1.0 \mathrm{mg} / \mathrm{dL})$ & 0.273 & $0.072-1.039$ & 0.0569 \\
\hline PT ( $\leq 80$ vs. $>80 \%)$ & 2.407 & $0.719-1.039$ & 0.1542 \\
\hline AST, U/L & 1.019 & $0.986-1.054$ & 0.260 \\
\hline ALT, U/L & 1.000 & $0.963-1.039$ & 0.995 \\
\hline $\mathrm{Hb}(\leq 13$ vs. $>13 \mathrm{~g} / \mathrm{dL})$ & 1.192 & $0.360-3.947$ & 0.7742 \\
\hline Platelets ( $\leq 8.5$ vs. $\left.>8.5 \times 10^{4} / \mu \mathrm{L}\right)$ & 1.262 & $0.319-3.947$ & 0.7403 \\
\hline NLR $(\leq 2.00$ vs. $>2.00)$ & 0.271 & $0.074-0.997$ & 0.050 \\
\hline $\mathrm{AFP}(\leq 400$ vs. $>400 \mathrm{ng} / \mathrm{mL})$ & 0.435 & $0.105-1.806$ & 0.2517 \\
\hline PIVKA-II ( $\leq 400$ vs. $>400 \mathrm{mAU} / \mathrm{mL})$ & 0.504 & $0.143-1.780$ & 0.2872 \\
\hline Initial dose of sorafenib & 0.998 & $0.995-1.001$ & 0.1902 \\
\hline Administration rate $(\leq 50$ vs. $>50 \%)$ & 0.875 & $0.253-3.023$ & 0.8325 \\
\hline Daily average dose ( $\leq 400$ vs. $>400 \mathrm{mg} /$ day) & 0.318 & $0.080-1.259$ & 0.1027 \\
\hline Times of additional treatments $(0,1$ vs. $\geq 2)$ & 4.794 & $1.167-19.687$ & 0.0296 \\
\hline
\end{tabular}

AST, aspartate aminotransferase; ALT, alanine aminotransferase; PT, prothrombin time; Hb, hemoglobin; NLR, neutrophil-to-lymphocyte ratio; AFP, alpha-feto protein; PIVKA-II, protein induced by vitamin-K absence-II; a Administration rate $=$ (dosing period of sorafenib/total treatment period after start of sorafenib) $\times 100$. 